\title{
Community-Based Risk Reduction in Zambia
}

\author{
D.L. Jones ${ }^{*}, 1$, S.M. Weiss ${ }^{1}$, D. Waldrop-Valverde ${ }^{1}$, N. Chitalu ${ }^{2}$, M. Mumbi and S. Vamos ${ }^{1}$ \\ ${ }^{I}$ Department of Psychiatry and Behavioral Sciences, University of Miami Miller School of Medicine, Miami, FL, USA \\ ${ }^{2}$ Department of Pediatrics, University of Zambia School of Medicine, Zambia
}

\begin{abstract}
Following the trial of a sexual risk reduction intervention conducted at the University Teaching Hospital (UTH) in Lusaka, Zambia, this pilot study sought to evaluate the feasibility of conducting the intervention at the Community Health Center (CHC) level. UTH staff implemented assessments and the intervention while CHC staff provided logistic and administrative support. HIV seropositive women $(\mathrm{CHC} n=200 ;$ UTH $n=612)$ attended group sessions in which male partners were randomized to a three-session or one-session group intervention arm. At baseline, consistent use of male and female condoms differed between sites (HIV+ UTH, 73\%, CHC, 88\%, HIV- UTH, 42\%, CHC 65\%); both sites increased combined condom use at 6 months post baseline and maintained increases over baseline at 12 months. Participants did not differ between sites at baseline on condom attitudes, HIV knowledge or self efficacy. At 12 months post baseline, both sites had improved in attitudes, knowledge and efficacy and participant retention was lower at the UTH site (77\% versus $82 \%$ ). Inconsistent sexual barrier users increased to consistent use at both sites after 6 months (HIV positive UTH, 96\%, CHC, 99\%, HIV negative UTH, 84\%, CHC 100\%). At 12 months, HIV negative CHC participants maintained higher levels of condom use in comparison with UTH participants $(F=7.17, p=.001)$. Results illustrate the feasibility and efficacy of conducting group sexual risk reduction interventions in the Zambian community, and the potential for the use of group interventions in conjunction with existing CHC Voluntary Counseling and Testing (VCT) programs.
\end{abstract}

\section{INTRODUCTION}

Sub-Saharan Africa is home to 24.7 million persons living with HIV [1]. Zambia, with a population of 10.2 million, has an estimated 1 million infections and a $17 \%$ national prevalence $[1,2]$ and recent health surveys indicate HIV prevalence is as high as $19.7 \%$ in urban areas in Zambia [3]. Current infection rates for women are higher than those for men $(54 \%$ of all infections are among women $[1,2]$ and the majority of HIV infection in women occurs in marital $(85 \%$ of all cases) and cohabiting relationships). Zambian youth have a $50 \%$ possibility of contracting HIV and dying of AIDS in the absence of treatment [2], but condom use may only temporarily increase following an HIV diagnosis. If both members of the couple do not receive VCT, the individual may not disclose their serostatus $[4,5]$ and protect an uninfected partner or restrict transmission of potentially resistant virus between infected partners [6]. Couples VCT (CVCT) has been the most successful HIV prevention approach for couples in Africa [7, 8], but strategies are needed to assess and maintain post CVCT risk reduction among large numbers of couples over time [9] at the community level.

In the capital city of Zambia, Lusaka, the twenty-seven urban Community Health Centres (CHCs) provide medical services to approximately two million residents and are the primary source of medical care and antiretroviral (ARV) medication distribution for the majority of HIV seropositive

*Address correspondence to this author at the University of Miami Miller School of Medicine, Dept. of Psychiatry \& Behavioral Sciences, 1400 NW $10^{\text {th }}$ Av., Miami, FL 33136, USA; Tel: 305243 2103; Fax: 305243 2126; E-mail: djones@med.miami.edu individuals in Zambia. Couples, herein defined as having a primary sexual relationship for more than six months, represent an important $\mathrm{CHC}$ population for behavioral intervention [10]. Sexual risk reduction in sero-concordant positive and sero-discordant relationships requires the agreement by both partners to long term adherence to sexual barrier use by male partners as the primary sexual decision makers [11, 12]. Condom use may be the most frequent outside the marital relationship and seen as intrusive within the marriage [13]. Our previous hospital-based clinical trial among seroconcordant and -discordant couples in Zambia successfully utilized a group sexual behavior intervention to enhance acceptability and the use of sexual barrier products [11] in HIV seropositive men [11] and positive and negative women [1416]. The intervention was designed to reduce sexual risk behavior and examined the relative impact of male involvement in the intervention on sexual risk behavior over time. This pilot study tested the feasibility of conducting the couples' intervention at the community health center (CHC) level, and contrasted the efficacy of the intervention among the CHC patients with the results obtained in the University Teaching Hospital (UTH)-based sample.

\section{MATERIALS AND METHODOLOGY}

Study design. The Partner Project was a randomized clinical trial that recruited 420 HIV seroconcordant positive and discordant Zambian couples 18 years of age or older between January 2003 and June 2006. Prior to study onset, Institutional Review Board and Research Ethics Committee approvals were obtained.

University Teaching Hospital (UTH) site. Study candidates participating at the UTH site were recruited from the 
hospital VCT, outlying CHCs and non-governmental organizations (NGOs). The UTH, co-located with the University of Zambia (UNZA) School of Medicine, is the largest public hospital in Lusaka. The hospital has an 1800 bed capacity with HIV-related conditions responsible for the majority of medical admissions. Of those attending the UTH VCT, our primary recruitment point, most were referred by the UTH and approximately $30 \%$ tested seropositive. Site offices were located within walking distance of the VCT.

Community Health Center (CHC) site. Study candidates at the $\mathrm{CHC}$ site were recruited from the M'Tendere $\mathrm{CHC}$ VCT. At the time of recruitment, M'Tendere CHC provided health services on contract with the Ministry of Health/Health Management Board to 92,440 patients, comprising 20,865 households. Medical services are provided to vulnerable populations (defined by income) through the community health waiver scheme, and all other patients pay a modest amount for services through a cost sharing scheme. The CHC provided administrative and logistic support, e.g., VCT referrals to the project staff, space to consent participants and hold intervention group sessions.

Participant intervention and examination protocol. Participants were screened for eligibility, i.e., verified couple relationship for a minimum of 6 months, sexually active in the last month, HIV seropositive or discordant, over 18 years of age, and provided individual informed consent prior to enrollment and then completed a baseline assessment. Female participants completed three sessions and their male partners were randomized to one of two conditions, comprising 1) female and male three-session or 2) female threesession and male one-session group intervention and were followed for 12 months (Fig. 1). Due to ethical considerations regarding the transmission of HIV by participants, no control group was included in this study. Data from previous studies [14] indicated that even enhanced "usual care" VCT clients significantly increased their use of sexual barrier products over time. The primary reason for ineligibility following referral was lack of sexual activity within the last month.

Recruiters, assessors and interventionists were multilingual and communicated in the participant's chosen dialect/language (Bemba, Nyanja, English). All assessment and intervention materials were translated into local language and back translated into English to verify accurate translation of meaning. Male and female participants were screened for STDs and penile/vaginal infections, notified of their STD results and provided with appropriate treatment prior to participation. Fifty-five cases of syphilis identified at baseline were identified and treated. All participants received monetary compensation. In the case of permanent loss of one member of a couple (e.g., illness, death, estrangement), individual participants were encouraged to continue to participate.

All female participants completed the multiple session intervention; their male partners were randomized into multiple session and single session group intervention arms to assess the relative influence of increased participation by male partners on sexual risk reduction within the couple.
Both arms were introduced to and provided with the same number of male and female condoms.

Intervention. The group intervention $(\mathrm{n}=10$ participants per gender specific group) was developed and standardized from feedback from studies with multicultural US and Zambian couples, and has been described previously [15]. The group emphasized participation and experimentation with sexual barrier products and provided an opportunity for practice, feedback, and reinforcement of sexual risk reduction strategies. The intervention utilized the conceptual model of the theory of reasoned action and planned behavior [17] applying cognitive behavioral strategies. Group facilitators were trained by US investigators and sessions were tape recorded and reviewed for quality assurance. Facilitators were provided with follow-up training and quality control throughout the course of the study.

Three \& One Session Intervention. Each of the two-hour sessions emphasized group cohesion and skill building in a supportive environment utilizing communication techniques, negotiation skills, and experiential/interactive skill training to expand and reframe perceptions of barrier use and to increase self-efficacy and skill mastery.

Session 1 included sexual risk reduction, HIV/STD transmission, skill training on male and female condom use and videos. Session 2 introduced vaginal lubricants (Astroglide $^{\circledR}$ and $\mathrm{KY}^{\circledR}$ gels, Lubrin ${ }^{\circledR}$ suppositories) as surrogates to assess acceptability for vaginal microbicides and included skill building and risk reduction strategies. Session 3 reinforced plans for risk avoidance and sexual communication strategies.

\section{Assessments}

Sexual Activities Questionnaire. This 55-item scale was adapted from the Sexual Risk Behavior Assessment Schedule [18]. Responses indicate the frequency of sexual intercourse (vaginal, oral, and anal) with primary partners (most frequent sexual relations) and non-primary partners (any other partners), sexual barrier product use, drug and alcohol use with sex and concomitant product use.

Sexual Diary. This scale assesses number of sex acts and number of uses of sexual barrier and lubricant products over the last 7 days.

Condom Attitudes. This 12 -item scale was includes items responded to on 4-point scales, $1=$ Strongly disagree, $4=$ Strongly agree. The scale is keyed so that higher scores represented more positive attitudes toward condoms. Zambian focus group feedback was used to adapt the scale to reflect local cultural norms.

AIDS Related Knowledge. This scale [19] uses 10 items to assess HIV risk and prevention-related knowledge. Items reflect information about HIV transmission, condom use, and AIDS-related knowledge and are responded to as Yes, No, or Don't Know. The AIDS knowledge test is scored for the number of correct responses.

Self Efficacy Questionnaire. This 9-item measure [20] assesses the level of sexual self-efficacy by asking participants to rate their level of comfort with specific sexual risk 

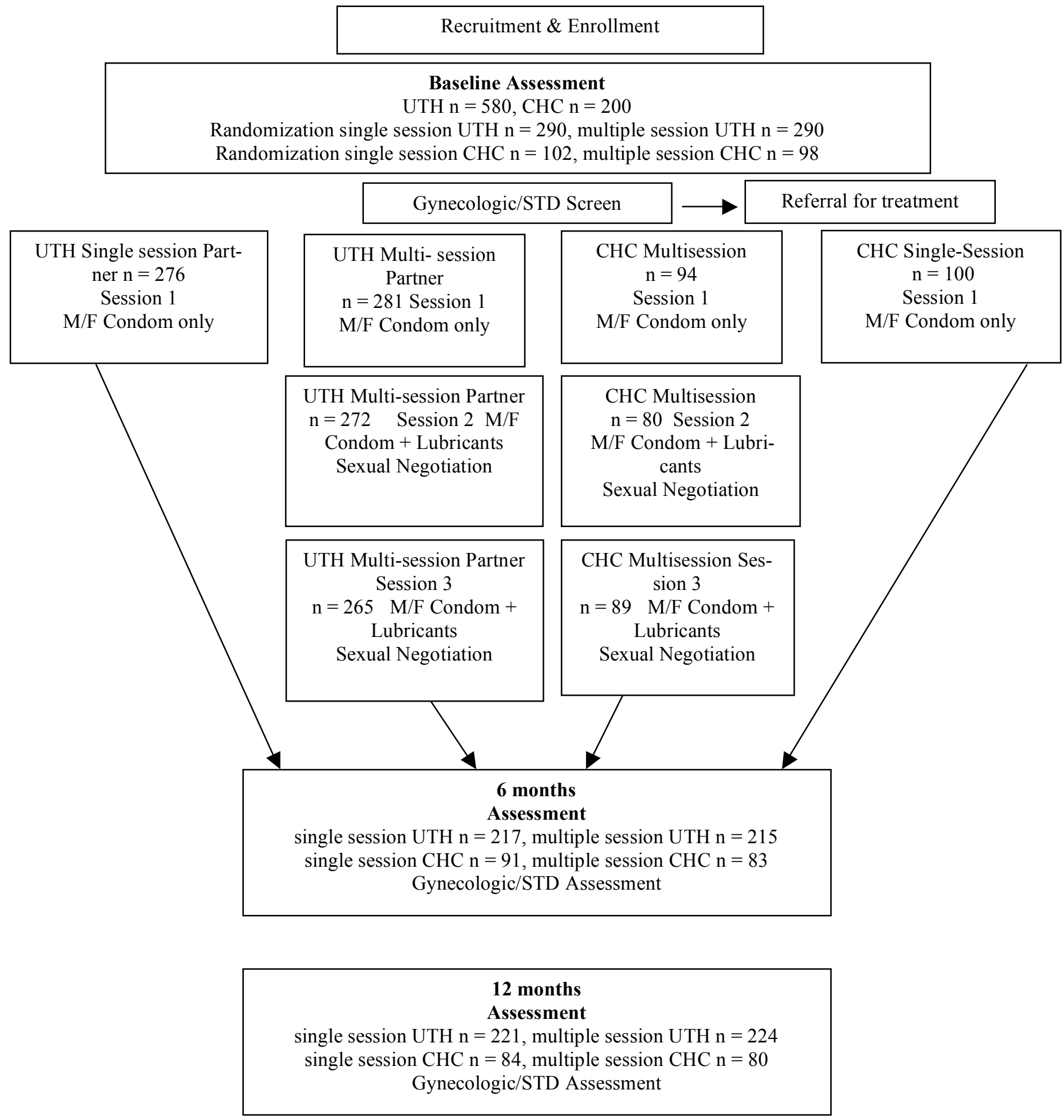

Fig. (1). Enrollment, randomization, intervention and assessment.

reduction behaviors using a Likert-like scale, including purchasing and using male or female condoms, refusing unprotected sex and negotiating condom use.

Statistical analyses. This study used a repeated measures design with experimental arm (three session, one session), site, (UTH, CHC), gender (male, female), and serostatus (positive, negative) as between-subjects factors and time (baseline and post-intervention, long term follow-up) as the within-subjects factor. Correlations are reported as Pearson's $r$ statistics; comparisons between factors and repeated measures are eported as $\mathrm{F}$ statistics using parametric tests as values were continuous with normal distributions. All comparisons used an alpha (2-tailed) of 0.05. Data was analyzed using the Statistical Package for Social Sciences (SPSS), Version 16. Differences between sites are noted in results, below. 
Table 1. Demographics by Site

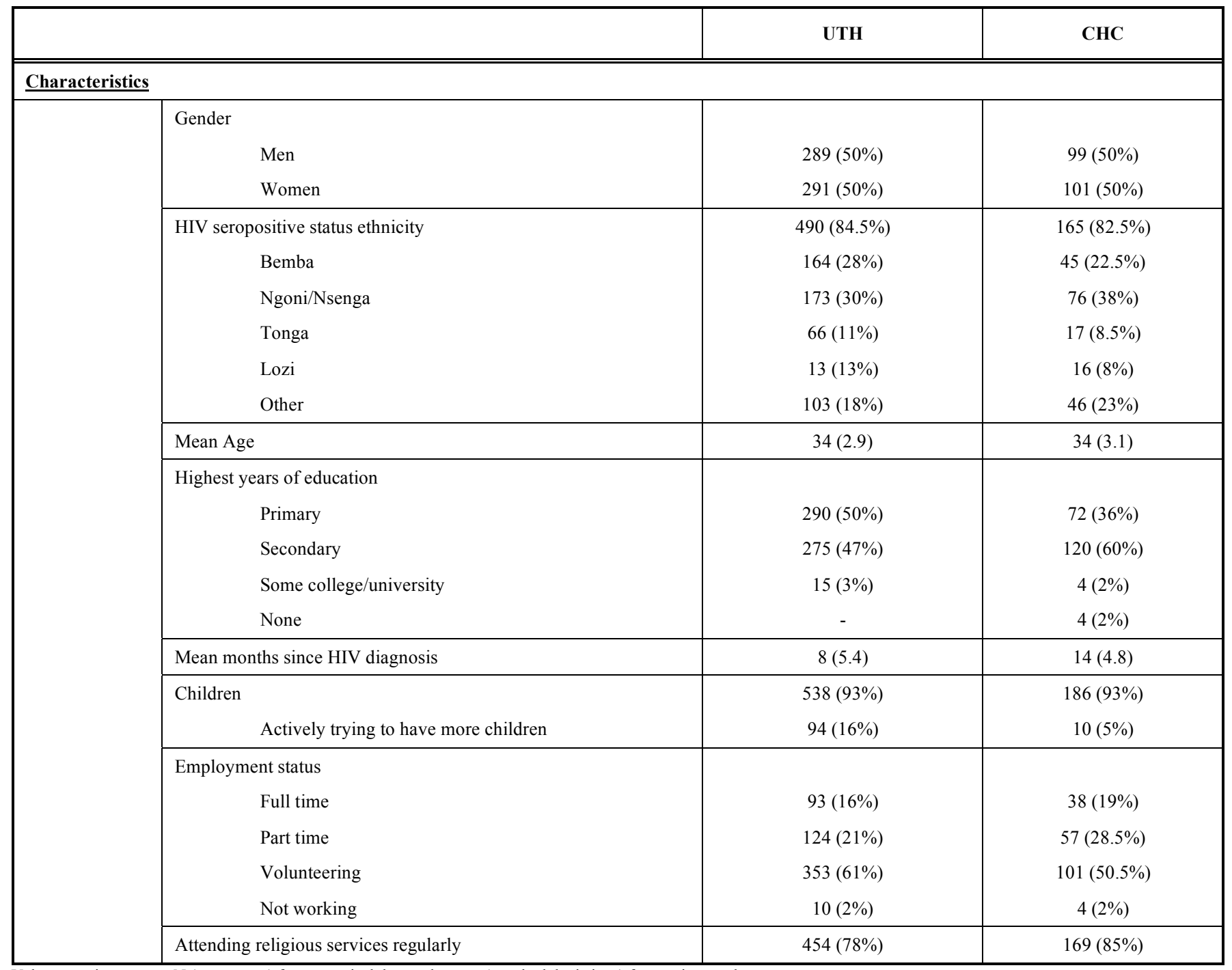

Values are given as raw $\mathrm{N}$ (percentage) for categorical data and means (standard deviations) for continuous data.

\section{RESULTS}

Sample demographic characteristics by gender and by site at baseline are presented in Table 1. Between sites, male participants differed with regard to employment status, education, ethnic group and current plans to have more children, and female participants differed with regard to employment status and education. Site participants differed regarding the mean length of time since diagnosis with HIV, $49 \%$ of the UTH and $40 \%$ of the CHC participants had been diagnosed in the last month, and use of male condoms did not differ statistically ( $p>0.05)$ at baseline, 6 or 12 months when controlling for serostatus. Participant retention was higher at the CHC site $(6$ months, $\mathrm{UTH},=87 \%, \mathrm{CHC}=74 \%, 12$ months, $\mathrm{UTH}=77 \%, \mathrm{CHC}=82 \%$ )

Data presented are by site, timepoint and serostatus, and do not include participants who reported no sexual activity in the last month at baseline, $7 \%$ of the sample at both sites (total both sites $\mathrm{n}=56$ ). Condom attitudes, HIV/AIDS related knowledge and sexual self efficacy are presented in Table 2; condom attitudes were more favorable at the $\mathrm{CHC}$ site than the UTH $(\mathrm{F}=60.2, \mathrm{p}<0.01)$.
Table 2. Assessment by Condition by Site at Baseline

\begin{tabular}{|l|c|c|}
\hline \multicolumn{1}{|c|}{ Baseline Measure } & UTH & CHC \\
\hline \hline $\begin{array}{l}\text { Previous experience with male } \\
\text { condom use } \\
\text { HIV + }\end{array}$ & & \\
HIV - & $446(84.5 \%)$ & $155(94 \%)$ \\
\hline $\begin{array}{l}\text { Previous experience with female } \\
\text { condom use }\end{array}$ & $64(71 \%)$ & $31(88.5 \%)$ \\
\hline $\begin{array}{l}\text { HIV + } \\
\text { HIV - }\end{array}$ & $131(25 \%)$ & $30(18 \%)$ \\
\hline $\begin{array}{l}\text { Consistent protected sex over 7 } \\
\text { day period } \\
\text { HIV + } \\
\text { HIV - }\end{array}$ & $12(13 \%)$ & $2(6 \%)$ \\
\hline Condom attitudes & $381(73 \%)$ & $145(88 \%)$ \\
\hline AIDS related knowledge & $38(42 \%)$ & $23(65 \%)$ \\
\hline Sexual self efficacy & $41.9(3.0)$ & $44.5(2.5)$ \\
\hline $\begin{array}{l}\text { Note: Values given are for raw N (percentages) for categorical data and means (stan- } \\
\text { dard deviations) for continuous variables. }\end{array}$ & $9.09(2.4)$ & $9.61(2.7)$ \\
\hline
\end{tabular}


Of those sampled, $48 \%$ of UTH participants and $80 \%$ of the CHC sample reported consistent male condom use in the last month, with $24 \%$ of the UTH sample and $9 \%$ of the $\mathrm{CHC}$ sample reporting male no condom use. However, overall, $57 \%$ of UTH participants and $71 \%$ of CHC participants, reported consistent male condom use over the last 7 days. Seronegative participants at both sites $(\mathrm{UTH}=90, \mathrm{CHC}=35)$ were less likely to use male condoms (UTH, $F=31.8, p<$ 0.001 , CHC, $F=6.46, p=0.012$ ).

\section{6 and 12 Months Post Baseline}

At 6 months post baseline, participants at both sites had improved their level of HIV/AIDS related knowledge, condom use attitudes and sexual self efficacy. Site and condition results are presented in Table $\mathbf{3}$; results are presented by individual site to highlight site specific outcomes. Consistent condom use increased at both sites, and among those identified as inconsistent condom users at baseline, use increased to similar levels (UTH 94\%; CHC 95\%). Seronegative participants increased consistent condom use at both sites (UTH, 84\%; CHC, 100\%). Both conditions had similar gains between sites among HIV seropositive participants (UTH three group condition, $95 \%$, single group, $97 \%, \mathrm{~F}=0.233$, $\mathrm{p}$ $=0.63$; CHC, three group condition, $100 \%$, single group, $99 \%, \mathrm{~F}=0.895, \mathrm{p}=0.357)$.

At 12 months post baseline, higher levels of condom attitudes, HIV knowledge and self efficacy were maintained at both sites. Levels of consistent condom use (UTH, 91\%; CHC, $100 \%$ ) were maintained by HIV seropositive participants at 12 months, but among seronegative participants at the UTH, levels decreased (62\%) from 6 months, while at the $\mathrm{CHC}$, seronegative participants maintained their increases $(100 \%)$.
Among inconsistent condoms users, initial gains were maintained and did not differ between sites (UTH, multiple group condition, 91\%, single group, 85, $\mathrm{F}=0.912, \mathrm{p}=0.343$; $\mathrm{CHC}$, multiple group condition, $100 \%$, single group, $89 \%, \mathrm{~F}=$ $0.766, \mathrm{p}=0.396$ ). Over 12 months, $\mathrm{CHC}$ seronegative participants maintained higher levels of sexual barrier use in comparison with UTH participants $(\mathrm{F}=7.17, \mathrm{p}=0.001)$.

\section{DISCUSSION}

This pilot study examined the feasibility of conducting group sexual risk reduction interventions in a Community Health Center setting and compared intervention outcomes with a clinical trial conducted at the University Teaching Hospital in Lusaka, Zambia. The study assessed sexual barrier use, condom use attitudes, HIV/AIDS knowledge, and sexual self efficacy among HIV seropositive and serodiscordant Zambian couples following a three session or single session intervention. Outcomes between both sites were similarly positive and support the use of group interventions in at the $\mathrm{CHC}$ level. Among inconsistent condom users, condom use increased over time, and among HIV seronegative participants, condom use was significantly higher at the $\mathrm{CHC}$ over the course of the intervention. Multiple and single session group interventions both reduced risk behavior.

Initial differences in condom use between the UTH and $\mathrm{CHC}$ patient populations may be due to several factors. Some patients receiving VCT at the UTH choose to test at the UTH due to perceived concerns about lack of confidentiality at the CHC, and may be less likely to accept and act upon their HIV seropositive diagnosis. Thus, the use of the UTH VCT services and subsequent recruitment at that site may also be associated with a denial of the implications of their HIV serostatus. Results may also reflect the use of

Table 3. Assessment by Condition by Site at $6 \& 12$ Months Post-Baseline

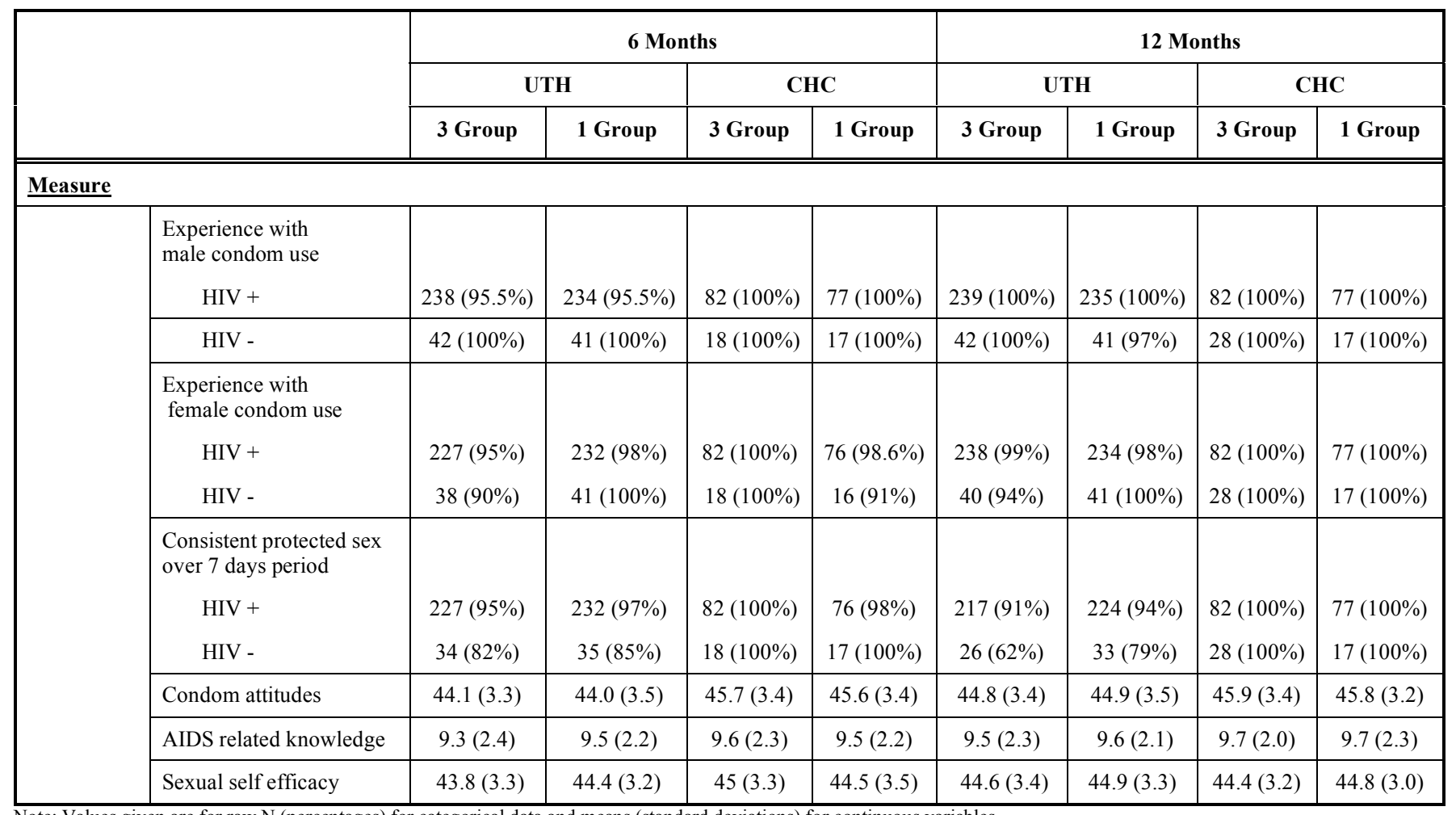


UTH interviewers for patient assessments at the $\mathrm{CHC}$, who may be provided a more confidential, and therefore, honest, assessment data. Study results may thus be limited by the use of UTH staff to provide the intervention at the $\mathrm{CHC}$ site.

Our previous research with HIV seropositive men and women in Zambia [11, 14-16] has found that group risk reduction interventions increased barrier use and this finding appears to have been sustained in the CHC population. As found in previous studies, results support the use of cognitive behavioral interventions to reduce the potential of HIV transmission to others among HIV seroconcordant individuals and discordant couples [12]. Interestingly, both multiple and single group interventions appeared to reduce risk in this population, which suggests that the single group intervention strategy may represent an effective brief, intervention which could be easily implemented at the community level.

The use of group interventions is both more efficient in terms of patients reached and reduction of staff burden. Traditional methods of encouraging sexual barrier use, such as providing condoms to those who have tested for HIV, differs from group interventions through the use of peers to provide information and motivational counseling. Groups enable patients to share their own experiences with sexual barrier products as well as provide a platform for sharing strategies for risk reduction. Group intervention strategies, such as the standardized methods used in this intervention, can be taught to community health center staff to encourage patients to learn skills for health behavior change while simultaneously enhancing the effectiveness of the health center in serving the needs of local populations.

Successful demonstration of the use of group strategies for patient education and skill building, with appropriate program evaluation, should be used to stimulate policy makers to consider promoting disease prevention by this potentially more effective use of limited health care resources. Ultimately, the process of translation of this intervention to the community context in Zambia will require a policy shift to group intervention strategies, and the uptake of the intervention by $\mathrm{CHCs}$ and the Ministry of Health; the current Ministry has already expressed interest in dissemination of successful programs such as the one outlined. First steps in implementation will include addressing issues such as buy-in by potentially overburdened administration and staff, training $\mathrm{CHC}$ healthcare and/or counseling personnel to implement and sustain the intervention, community advisory board involvement, identification of funding lines for intervention supplies and staff supervision and training, etc. Currently, this team is actively exploring these translation issues at additional CHCs and community advisory boards in urban Lusaka as well as policy issues associated with their implementation.

Existing studies of VCT in Zambia have focused exclusively on individual and couples based counseling strategies [10] and presently, VCT is conducted singly or in couples. Single or multiple group interventions will enable counselors or healthcare staff to provide information and guidance to larger numbers of people who then receive mutual support following an HIV diagnosis. The relative disparity in condom use at baseline between those who are HIV seropositive and those who are seronegative, given that both groups have seropositive partners, also highlights the importance of intervention in both infected and at-risk populations. The application of these types of group intervention strategies in conjunction with existing Voluntary Counseling and Testing programs would provide a valuable addition to individual and population focused prevention efforts in the Zambian context.

\section{ACKNOWLEDGEMENTS}

This research was made possible by a grant from the National Institute of Child Health and Human Development, R24HD43613. We would like to acknowledge the members of our research teams at the University Of Zambia School Of Medicine, the University Teaching Hospital in Lusaka, community sites providing referrals in Zambia and our study participants.

\section{REFERENCES}

[1] UNAIDS. UNAIDS/WHO AIDS Epidemic update 2006 [Retrieved May 31, 2007]. Available from: http://www.unaids.org/en/HIV_ data/epi 2006/

[2] Ministry of Health, Central Board of Health, Government Republic of Zambia. Zambia Antenatal Clinic Sentinel Surveillance Report, 1994-2004. Lusaka: Government Printing Office 2005.

[3] Few Zambians are accessing VCT services 2008 [Retrieved July 3, 2008]. Available from http://www.lusakatimes.com/?p=3119

[4] Simbayi LC, Kalichman SC, Strebel A, et al. Disclosure of HIV status to sex partners and sexual risk behaviours among HIVpositive men and women, Cape Town, South Africa. Sex Transm Infect 2007; 83(1): 29-34.

[5] Schatz E. 'Take your mat and go!': Rural Malawian women's strategies in the HIV/AIDS era. Cult Health Sex 2005; 7: 479-92.

[6] Kalichman S. "The synergies of HIV treatment, adherence and prevention". $2^{\text {nd }}$ International Conference on HIV Treatment Adherence: Jersey City, March 28-30, 2007.

[7] Chomba E, Allen S, Kanweka W, et al., the Rwanda Zambia HIV Research Group. Evolution of couples' voluntary counseling and testing for HIV in Lusaka, Zambia. J Acquir Immune Defic Syndr 2007; 47(1): 108-15.

[8] Roth DL, Stewart KE, Clay OJ, et al. Sexual practices of HIV discordant couples in Rwanda: Effects of a testing and counseling program for men. Int J STD AIDS 2001; 12: 181-8.

[9] Bunnell RE, Nassozi J, Marum E, et al. Living with discordance: Knowledge, challenges and prevention strategies of HIVdiscordant couples in Uganda. AIDS Care 2005; 17: 999-1012.

[10] Allen S, Meinzen-Derr J, Kautzman M, et al. Sexual behavior of HIV discordant couples after HIV counseling and testing. AIDS 2003; 17(5): 733-40.

[11] Jones D, Weiss SM, Chitalu N, et al. Acceptability and use of sexual barrier products and lubricants among HIV seropositive Zambian men. AIDS Patient Care ST; (in press).

[12] The Healthy Living Project Team. Effects of a behavioral intervention to reduce risk of transmission among people living with HIV: The healthy living project randomized controlled study. J Acquir Immune Defic Syndr 2007; 44(2): 213-21.

[13] Chimbiri AM. The condom is an "intruder" in marriage: Evidence from rural Malawi. Soc Sci Med 2007; 64: 1102-15.

[14] Jones DL, Weiss SM, Bhat GJ, et al. A sexual barrier intervention for HIV+/- Zambian women: Acceptability and use of vaginal chemical barriers. J Multicult Nurs Health 2004; 10: 27-31.

[15] Jones DL, Bhat GJ, Weiss SM, et al. Influencing sexual practices among HIV positive Zambian women. AIDS Care 2006; 18(6): 629-34.

[16] Jones DL, Weiss SM, Chitalu N, Bwalya V, Villar O. Acceptability of microbicidal surrogates among Zambian women. Sex Transm Dis 2008; 35(2): 147-53. 
[17] Albarracin D, Johnson BT, Fishbein M, et al. Theories of reasoned action and planned behavior as models of condom use: A metaanalysis. Psych Bull 2001; 127: 142-61.

[18] Meyer-Bahlberg HFL, Ehrhardt AA, Exner TM, et al. Sexual risk behavior assessment schedule: Adult (SERBAS-A-DF-4) manual. 1990.
[19] Kalichman SC. HIV-AIDS: Prevalence, predictors, and emerging clinical interventions. Clin Psychol: Sci Prac 2000; 7: 32-47.

[20] Smith KW, McGraw SA, Costa LA, McKinlay JB. A self-efficacy scale for HIV risk behaviors: Development and evaluation. AIDS Educ Prev 1996; 8: 97-105.

(C) Jones et al.; Licensee Bentham Open.

This is an open access article licensed under the terms of the Creative Commons Attribution Non-Commercial License (http://creativecommons.org/licenses/by$\mathrm{nc} / 3.0 /$ / / which permits unrestricted, non-commercial use, distribution and reproduction in any medium, provided the work is properly cited. 